\title{
Inexactness of SDP Relaxation and Valid Inequalities for Optimal Power Flow
}

\author{
Burak Kocuk, Santanu S. Dey, X. Andy Sun *
}

December 19, 2014

\begin{abstract}
It has been recently proven that the semidefinite programming (SDP) relaxation of the optimal power flow problem over radial networks is exact under technical conditions such as not including generation lower bounds or allowing load over-satisfaction. In this paper, we investigate the situation where generation lower bounds are present. We show that even for a two-bus one-generator system, the SDP relaxation can have all possible approximation outcomes, that is (1) SDP relaxation may be exact or (2) SDP relaxation may be inexact or (3) SDP relaxation may be feasible while the OPF instance may be infeasible. We provide a complete characterization of when these three approximation outcomes occur and an analytical expression of the resulting optimality gap for this two-bus system. In order to facilitate further research, we design a library of instances over radial networks in which the SDP relaxation has positive optimality gap. Finally, we propose valid inequalities and variable bound tightening techniques that significantly improve the computational performance of a global optimization solver. Our work demonstrates the need of developing efficient global optimization methods for the solution of OPF even in the simple but fundamental case of radial networks.
\end{abstract}

\section{Introduction}

Optimal Power Flow (OPF) was first introduced in the 1960s [5] and much effort has been devoted to its solution, which has resulted in a rich literature. Roughly speaking, we can categorize the previous work into three categories.

${ }^{*}$ The authors are with the School of Industrial and Systems Engineering, Georgia Institute of Technology, 765 Ferst Drive, NW Atlanta, Georgia 30332-0205 (e-mail: burak.kocuk, santanu.dey, andy.sun@isye.gatech.edu). 
The first category of algorithms find local optimal solutions or stationary points using optimization procedures such as interior point methods (e.g. MATPOWER [27]). The shortcoming of these local methods is that if a solution is obtained, there is no guarantee for global optimality or even any evidence of how good this solution is. For instance, in [4], there are several examples which have multiple local optima and it has been shown that local solvers tend to converge to the solution which is closest to the initial guess.

The second category of algorithms attempt to obtain global optimal solutions of OPF by solving convex relaxations. For instance, one popular approach is to use semidefinite programming (SDP) relaxations of the original OPF problem. Since SDPs are polynomially solvable, this method suggests that OPF can be solved efficiently provided that the relaxation is exact, i.e., the SDP relaxation finds the global optimal solution for the OPF problem. A recent, comprehensive survey can be found in $[16,17]$.

- One of the early works that popularizes this approach is [14]. It is shown that the SDP relaxation is tight for a resistive network with no reactive loads where demand oversatisfaction is allowed, as long as the dual variables are positive. It was conjectured that under normal operating conditions the SDP relaxation is tight.

- However, [15] gives a very simple counterexample (a 3-bus cycle) with nonzero optimality gap.

- In [20], it is proven that SDP relaxation is exact if load over-satisfaction is allowed and a sufficient number of virtual phase shifters are present.

- An attempt to solve OPF using SDP relaxation is made in [26] for radial networks. In this work, it is proven that under operational constraints on voltage magnitudes, line losses, and line flows, the SDP relaxation is tight if there are no lower bounds on real and reactive power generation at any bus. Similar results are also obtained in $[3,2]$ without line limit constraints.

- In [13], it is proven that if voltage magnitudes are fixed, then the convex relaxations are tight under practical angle restrictions for radial networks in the presence of only real power lower bounds. This result extends to the case with variable voltage magnitudes under reasonable assumptions.

As we observe above, the exactness of the SDP relaxation can only be guaranteed for special classes of OPF instances, often when we disregard some generation lower bounds. Unfortunately, if the SDP relaxation is not tight, the physical meaning of its solution is not easy to recover. In this case, an alternative approach would be to use an hierarchy for SDPs 
[12] as suggested in [11]. However, this approach may not be practical as the size of the SDPs grows larger with the order of the relaxation.

The third category of algorithms attempt to remove the pitfalls of the previous two approaches by endeavoring to obtain globally optimal solutions. One such algorithm based on branch-and-bound method is proposed in [19] for the solution of OPF. Lagrangian relaxation is used to find lower bounds while a local solver (IPOPT) is utilized to obtain upper bounds. Global solution techniques are in their infancy today and much work needs to be done to make them practically efficient.

In this paper, we focus on the OPF problem on radial networks in the presence of generation lower bounds on both real and reactive power. The goal of this paper is two fold: To highlight the inexactness of standard convex relaxations for these instances and to make algorithmic progress in solving such instances globally. We make two comments here in relation to the class of OPF problems we consider and our assumptions. First, although most power flow networks are not radial, they are usually quite sparse and analyzing radial networks can therefore be beneficial for their own right, especially in the case of distribution networks [13]. Second, typically power systems have ramping constraints, so that the power generation in the next time period cannot deviate from the current one too much. Hence, it is important to make a study of the effects of lower bounds.

In practice, SDPs may become prohibitively expensive as the size of the network grows larger. One can turn to second order conic programming (SOCP) relaxations, which are in general weaker than their SDP counterparts. However, in [21], it has been proven that both types of relaxations give the same lower bound for the OPF problem over radial networks even if they are inexact. Therefore any result stated for SOCPs in this paper holds for SDP relaxations and vice-versa.

The rest of the paper is organized as follows: In the next section, we review the rectangular formulation of the OPF problem and a reformulation that leads to the SOCP relaxation. In Section 3, we begin working on our first goal by providing a complete characterization of the approximation performance of SOCP relaxation for a two-bus system. In Section 4, we further study the feasible regions of two small systems. Then, in Section 5, we begin working on our second goal by providing a library of radial network instances generated from MATPOWER test cases for which SOCP relaxation is inexact. In Section 6, we propose valid inequalities for the SOCP relaxation, which significantly improves the computational performance of a global solver. Concluding remarks are made in Section 7. 


\section{Optimal Power Flow}

Consider a typical power network, where $\mathcal{B}, \mathcal{G}$ and $\mathcal{L}$ denote respectively the set of buses, generators and transmission lines. The nodal admittance matrix $Y \in \mathbb{C}^{|\mathcal{B}| \times|\mathcal{B}|}$ has component $Y_{i j}=G_{i j}+\mathrm{i} B_{i j}$ for each line $(i, j)$ and $G_{i i}=g_{i i}-\sum_{j \neq i} G_{i j}, B_{i i}=b_{i i}-\sum_{j \neq i} B_{i j}$, where $g_{i i}$ (resp. $b_{i i}$ ) is the shunt conductance (resp. susceptance) at bus $i$. Let $p_{i}^{g}, q_{i}^{g}$ (resp. $p_{i}^{d}, q_{i}^{d}$ ) be the real and reactive power output of the generator (resp. load) at bus $i$. The complex voltage $V_{i}$ at bus $i$ can be expressed either in the rectangular form as $V_{i}=e_{i}+\mathrm{i} f_{i}$ or in the polar form as $V_{i}=\left|V_{i}\right|\left(\cos \theta_{i}+\mathrm{i} \sin \theta_{i}\right)$, where the voltage magnitude $\left|V_{i}\right|^{2}=e_{i}^{2}+f_{i}^{2}$.

The OPF problem in the rectangular form is given as

$$
\begin{array}{ll}
\min & \sum_{i \in \mathcal{G}} C_{i}\left(p_{i}^{g}\right) \\
\text { s.t. } & p_{i}^{g}-p_{i}^{d}=\sum_{j \in \delta(i)}\left[G_{i j}\left(e_{i} e_{j}+f_{i} f_{j}\right)-B_{i j}\left(e_{i} f_{j}-e_{j} f_{i}\right)\right] \\
& i \in \mathcal{B} \\
& \\
q_{i}^{g}-q_{i}^{d}=\sum_{j \in \delta(i)}\left[-B_{i j}\left(e_{i} e_{j}+f_{i} f_{j}\right)-G_{i j}\left(e_{i} f_{j}-e_{j} f_{i}\right)\right] \\
\left(V_{i}^{\text {min }}\right)^{2} \leq e_{i}^{2}+f_{i}^{2} \leq\left(V_{i}^{\max }\right)^{2} \quad i \in \mathcal{B} \quad \\
p_{i}^{\min } \leq p_{i}^{g} \leq p_{i}^{\max } \quad i \in \mathcal{B} \\
q_{i}^{\min } \leq q_{i}^{g} \leq q_{i}^{\max } \quad i \in \mathcal{B} \\
\end{array}
$$

Here $C_{i}\left(p_{i}^{g}\right)$ in (1a) represents the production cost of generator $i$, which typically is either a linear or a convex quadratic nondecreasing function of $p_{i}^{g}$. Constraints (1b)-(1c) enforce flow conservation at each bus $i$, where $\delta(i)$ is the set of buses adjacent to $i$ and including $i$. Constraint (1d) limits the upper and lower bounds on the bus voltage magnitudes. Usually $V_{i}^{\min }$ and $V_{i}^{\max }$ are close to the unit voltage. Constraints (1e)-(1f) are the upper and lower bounds on generator $i$ 's real and reactive power, respectively. Here, we have $p_{i}^{\min }=p_{i}^{\max }=$ $q_{i}^{\min }=q_{i}^{\max }=0$ for bus $i$ where there is no generator, i.e. $i \in \mathcal{B} \backslash \mathcal{G}$.

One can equivalently formulate the above OPF problem in polar coordinates. Sometimes, the rectangular formulation is preferred since the Hessian matrix of the constraints is constant and this is an advantage for the interior point methods. On the other hand, when the voltage magnitude is fixed at some buses, the polar formulation may become more advantageous [22]. 
We can observe that all the nonlinearities in (1) are of the following three types:

$$
\text { (1) } e_{i}^{2}+f_{i}^{2} \quad(2) e_{i} e_{j}+f_{i} f_{j} \quad(3) e_{i} f_{j}-e_{j} f_{i}
$$

which are equal to $\left|V_{i}\right|^{2},\left|V_{i}\right|\left|V_{j}\right| \cos \left(\theta_{i}-\theta_{j}\right)$, and $\left|V_{i}\right|\left|V_{j}\right| \sin \left(\theta_{i}-\theta_{j}\right)$ in the polar form, respectively. Let us define new variables $c_{i i}, c_{i j}$, and $s_{i j}$ for each of these three quantities. Since the cosine function is even and the sine function is odd, we also have $c_{i j}=c_{j i}$ and $s_{i j}=-s_{j i}$. On each line $(i, j)$, these quantities are linked through the fundamental trigonometric identity $\cos ^{2}\left(\theta_{i}-\theta_{j}\right)+\sin ^{2}\left(\theta_{i}-\theta_{j}\right)=1$, which translates into

$$
\left(e_{i} e_{j}+f_{i} f_{j}\right)^{2}+\left(e_{i} f_{j}-e_{j} f_{i}\right)^{2}=\left(e_{i}^{2}+f_{i}^{2}\right)\left(e_{j}^{2}+f_{j}^{2}\right)
$$

in the rectangular form. In the space of our new variables, this relation is expressed in the following quadratic equation $c_{i j}^{2}+s_{i j}^{2}=c_{i i} c_{j j}$, which describes the surface of a rotated second-order cone in four dimensions.

Now, we are ready to reformulate OPF using this idea:

$$
\begin{aligned}
& \min \sum_{i \in \mathcal{G}} C_{i}\left(p_{i}^{g}\right) \\
& \text { s.t. } \quad p_{i}^{g}-p_{i}^{d}=\sum_{j \in \delta(i)}\left[G_{i j} c_{i j}-B_{i j} s_{i j}\right] \quad i \in \mathcal{B} \\
& q_{i}^{g}-q_{i}^{d}=\sum_{j \in \delta(i)}\left[-B_{i j} c_{i j}-G_{i j} s_{i j}\right] \quad i \in \mathcal{B} \\
& \left(V_{i}^{\min }\right)^{2} \leq c_{i i} \leq\left(V_{i}^{\max }\right)^{2} \quad i \in \mathcal{B} \\
& p_{i}^{\min } \leq p_{i}^{g} \leq p_{i}^{\max } \quad i \in \mathcal{B} \\
& q_{i}^{\min } \leq q_{i}^{g} \leq q_{i}^{\max } \quad i \in \mathcal{B} \\
& c_{i j}=c_{j i} \quad(i, j) \in \mathcal{L} \\
& s_{i j}=-s_{j i} \quad(i, j) \in \mathcal{L} \\
& c_{i j}^{2}+s_{i j}^{2}=c_{i i} c_{j j} . \quad(i, j) \in \mathcal{L}
\end{aligned}
$$

This reformulation (2) is exact for any radial network, because the following equations on voltage angles

$$
\begin{aligned}
\sin \left(\theta_{i}-\theta_{j}\right)=\frac{s_{i j}}{\sqrt{c_{i i} c_{j j}}}, & (i, j) \in \mathcal{L} \\
\cos \left(\theta_{i}-\theta_{j}\right)=\frac{c_{i j}}{\sqrt{c_{i i} c_{j j}}}, & (i, j) \in \mathcal{L}
\end{aligned}
$$


have a unique solution as long as the underlying network is radial, where $s_{i j}, c_{i j}$ are obtained from solving (2). An alternative proof can be seen in [7]. For meshed networks, however, the reformulation (2) is exact only if we include (3)-(4) in the constraints. This idea is first proposed to solve the load flow problem for radial and meshed networks $[6,8,9]$. Then, it is adapted to OPF in [10].

Except the coupling constraints (2i), all other constraints in (2) are now linear. Hence, all the nonconvexity of the OPF problem (1) in a radial network is captured by (2i), and the feasible region is the intersection of a polytope defined by $(2 \mathrm{~b})-(2 \mathrm{~h})$ with the boundaries of rotated second-order cones defined by $(2 \mathrm{i})$. It is straightforward to obtain a second-order cone programming (SOCP) relaxation of (2) by relaxing constraint (2i) as follows:

$$
c_{i j}^{2}+s_{i j}^{2} \leq c_{i i} c_{j j} \quad(i, j) \in \mathcal{L}
$$

which can be written more explicitly as a SOCP constraint:

$$
c_{i j}^{2}+s_{i j}^{2}+\left(\frac{c_{i i}-c_{j j}}{2}\right)^{2} \leq\left(\frac{c_{i i}+c_{j j}}{2}\right)^{2} \quad(i, j) \in \mathcal{L} .
$$

The SOCP relaxation is defined as $(2 \mathrm{a})-(2 \mathrm{~h})$ and (6). It is proven that in radial networks the SOCP relaxation is equivalent to the SDP relaxation [21]. In this paper, we focus on SOCP relaxation due to its superior computational performance.

\section{Analytical Study of a Two-Bus System}

In this section, we study the two-bus system with one generator and one load. This is arguably the simplest power system, but also one of the most fundamental models in power system analysis. Surprisingly, for this simple system, the SOCP relaxation with generation lower bounds can have all three possible outcomes in terms of optimality gap, namely (1) SOCP obtains exact solution (i.e. optimality gap is zero); (2) SOCP is feasible, yet OPF is infeasible (optimality gap is infinite); (3) SOCP has a finite optimality gap, and we give an analytical expression of this gap. We identify parameter ranges in closed form for each of these outcomes. We also study the feasible region projected in the space of squared bus voltage magnitudes to gain geometric intuition.

Let us assume that bus 1 is a generator bus and bus 2 is a load bus. Further assume that $g_{i i}=b_{i i}=0$ and $G:=G_{12}<0$ and $B:=B_{12}>0$ (the analysis for $B<0$ is similar). Also assume the production cost $C_{1}\left(p_{1}^{g}\right)$ is linear in $p_{1}^{g}$. 
Figure 1: Projection of feasible region of 2-bus, 1-generator examples onto $\left(c_{11}, c_{22}\right)$ space for five cases. Horizontal axis is $c_{11}$ and vertical axis is $c_{22}$. Solid black curve is (10) containing the feasible region of OPF with dashed lines being two asymptotes shown in Fig. 1a. Green and red lines are bounds on $c_{11}$ and $c_{22}$, resp. Magenta line is the effective lower bound on $c_{11}-c_{22}$. Blue region is the feasible region of SOCP relaxation. All figures are in p.u.

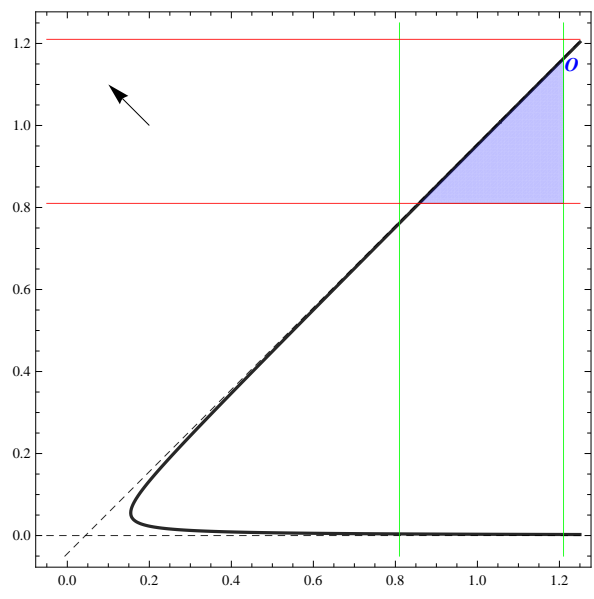

(a) Case 1: SOCP relaxation is exact.

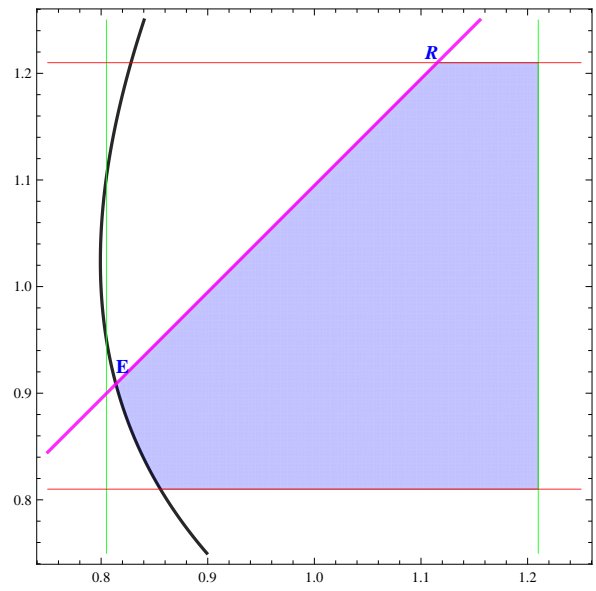

(c) Case 3: SOCP relaxation is exact.

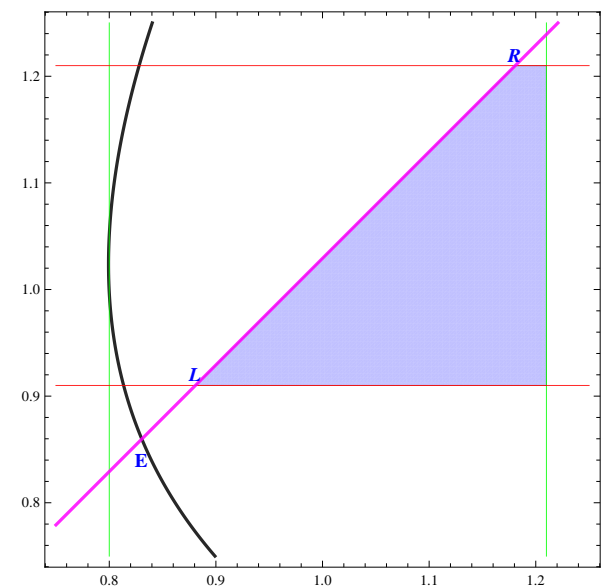

(b) Case 2: SOCP relaxation is feasible, $\mathrm{OPF}$ is infeasible.

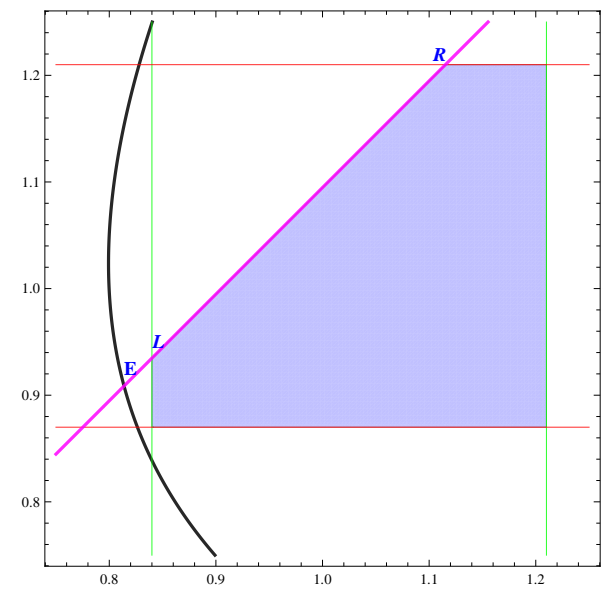

(d) Case 4: SOCP relaxation is feasible, $\mathrm{OPF}$ is infeasible.

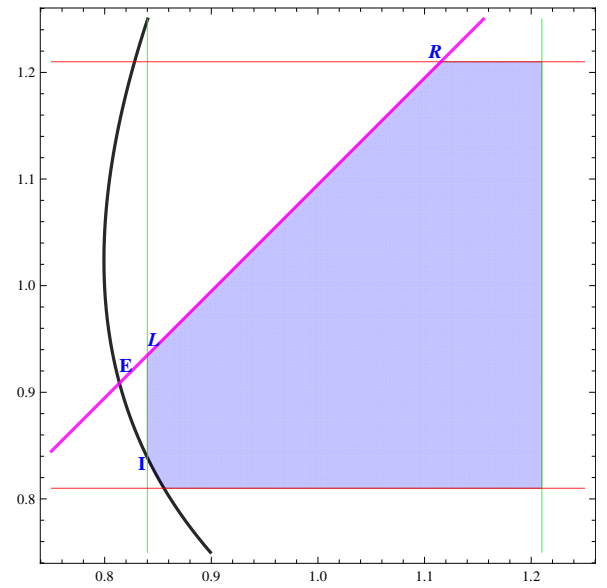

(e) Case 5: SOCP relaxation is inexact. 


\subsection{Feasible Region Projected to $\left(c_{11}, c_{22}\right)$ space}

The linear equality system (2b)-(2c) can be written as

$$
\left[\begin{array}{rrrrrr}
1 & & G & & -G & B \\
& 1 & -B & & B & G \\
& & & G & -G & -B \\
& & & -B & B & -G
\end{array}\right]\left[\begin{array}{c}
p_{1}^{g} \\
q_{1}^{g} \\
c_{11} \\
c_{22} \\
c_{12} \\
s_{12}
\end{array}\right]=\left[\begin{array}{c}
0 \\
0 \\
p_{2}^{d} \\
q_{2}^{d}
\end{array}\right]
$$

Let us define

$$
\alpha:=\frac{B p_{2}^{d}+G q_{2}^{d}}{B^{2}+G^{2}} \text { and } \beta:=\frac{G p_{2}^{d}-B q_{2}^{d}}{B^{2}+G^{2}}
$$

which are constant for fixed $B, G$ and load. Solving the linear system (7), we can express $\left(p_{1}^{g}, q_{1}^{g}, c_{12}, s_{12}\right)$ in terms of $\left(c_{11}, c_{22}\right)$ as follows

$$
\begin{aligned}
s_{12} & =-\alpha \\
c_{12} & =c_{22}-\beta \\
p_{1}^{g} & =-G\left(c_{11}-c_{22}\right)-G \beta+B \alpha \\
q_{1}^{g} & =B\left(c_{11}-c_{22}\right)+B \beta+G \alpha .
\end{aligned}
$$

We now reformulate constraint (2i) using (9a) and (9b) as

$$
\left(c_{22}-\beta\right)^{2}+\alpha^{2}=c_{11} c_{22} \Rightarrow c_{11}=c_{22}-2 \beta+\frac{\alpha^{2}+\beta^{2}}{c_{22}}
$$

which defines a hyperbola for $\left(c_{11}, c_{22}\right)$ with two asymptotes: $c_{11}-c_{22}=-2 \beta$ and $c_{22}=0$.

Observe that this hyperbola together with the constraints on $c_{11}$ and $c_{22}$ implied from (2d)-(2f) define the feasible region of the OPF problem projected to the $\left(c_{11}, c_{22}\right)$ space. In particular, (2d) impose a box constraint on $c_{11}$ and $c_{22}$, whereas (2e)-(2f) imply upper and lower bounds on the difference $c_{11}-c_{22}$, which defines a region parallel to the first asymptote $c_{11}-c_{22}=-2 \beta$. Figure 1a depicts the entire feasible regions of OPF in black curve and of SOCP relaxation in the blue region. Figures 1b-1e zoom in particular parts.

Furthermore, since the objective function $C_{1}\left(p_{1}^{g}\right)$ is assumed to be linear in $p_{1}^{g}$ and by $(9 \mathrm{c})$, we can see that the level set of the objective function in $\left(c_{11}, c_{22}\right)$ is also parallel to the first asymptote, and decreases toward the upper left corner as pointed by the arrow in Figure 1. Therefore, only the lower bounds on $p_{1}^{g}$ and $q_{1}^{g}$ can affect the optimal solution of 
(2). For this reason, we find the effective lower bound for the difference $c_{11}-c_{22}$ as

$$
\Delta=\max \left\{\frac{p_{1}^{\min }+G \beta-B \alpha}{-G}, \frac{q_{1}^{\min }-B \beta-G \alpha}{B}\right\},
$$

which is given by the lower bounds of (2e)-(2f), and is plotted as magenta lines in Figure 1. Also note that as $p_{1}^{\min }$ and $q_{1}^{\mathrm{min}}$ increase, the line $c_{11}-c_{22} \geq \Delta$ moves toward the lower right corner in Figure 1.

\subsection{Complete Characterization of Approximation Outcomes}

At this point, we are ready to explore the optimal solutions of the OPF (2) and its SOCP relaxation and classify all five possible cases of the configurations of their feasible regions and the associated approximation outcomes.

- First of all, let us assume that $\Delta$ defined in (11) is small enough. In this case, as depicted in Figure 1a, the optimal solution of both the OPF and the SOCP is unique and given by

$$
\left(c_{11}^{O}, c_{22}^{O}\right)=\left\{\begin{array}{l}
\left(\bar{c}_{22}-2 \beta+\frac{\alpha^{2}+\beta^{2}}{\bar{c}_{22}}, \bar{c}_{22}\right) \quad \text { if }(\mathrm{a}) \text { holds } \\
\left(\bar{c}_{11}, \frac{2 \beta+\bar{c}_{11}+\sqrt{\left(2 \beta+\bar{c}_{11}\right)^{2}-4\left(\alpha^{2}+\beta^{2}\right)}}{2}\right) \text { o.w. }
\end{array}\right.
$$

where condition (a) is $\left(\bar{c}_{22}-\beta\right)^{2}+\alpha^{2} \leq \bar{c}_{11} \bar{c}_{22}$ and $\bar{c}_{i i}:=\left(V_{i}^{\max }\right)^{2}$. Hence, the SOCP relaxation is exact. This result is in accordance with the results in [26].

- Consider the case where $\Delta$ is large enough. In particular, $c_{11}^{O}-c_{22}^{O}<\Delta$. Define the intersection of $c_{11}-c_{22}=\Delta$ with the binding upper bound of either $c_{11}$ or $c_{22}$ as

$$
\left(c_{11}^{R}, c_{22}^{R}\right)=\left\{\begin{array}{l}
\left(\bar{c}_{22}+\Delta, \bar{c}_{22}\right) \text { if } \bar{c}_{11}-\bar{c}_{22} \geq \Delta \\
\left(\bar{c}_{11}, \bar{c}_{11}-\Delta\right) \text { o.w. }
\end{array}\right.
$$

Note that this point is OPF infeasible despite being SOCP optimal. Next, define the intersection of the hyperbola (10) and $c_{11}-c_{22}=\Delta$ as

$$
\left(c_{11}^{E}, c_{22}^{E}\right)=\left(\frac{\alpha^{2}+\beta^{2}}{2 \beta+\Delta}+\Delta, \frac{\alpha^{2}+\beta^{2}}{2 \beta+\Delta}\right)
$$

If $c_{22}^{E}<\underline{c}_{22}$, where $\underline{c}_{i i}:=\left(V_{i}^{\text {min }}\right)^{2}$, then SOCP is feasible while OPF is infeasible. An example of this case can be seen from Figure 1b, which shows the zoomed in part of the hyperbola. 
Figure 2: Projection of feasible region of 2-bus, 2-generator example onto $\left(p_{1}^{g}, p_{2}^{g}\right)$ space. Horizontal axis is $p_{1}^{g}$ and vertical axis is $p_{2}^{g}$. Black curve is an ellipse with counterclockwise orientation that contains the feasible region of OPF problem whereas blue region is its SOCP relaxation. Green lines are the lower bound on $p_{1}^{g}$ and $p_{2}^{g}$ while red lines are the lower bound on $q_{1}^{g}$ and $q_{2}^{g}$. Dashed lines represent angle bounds corresponding to $30^{\circ}$. Assuming linear functions, the arrow shows the cost vector. Blue and orange dots are respectively the optimal solutions of SOCP relaxation and OPF, whenever the latter exists.

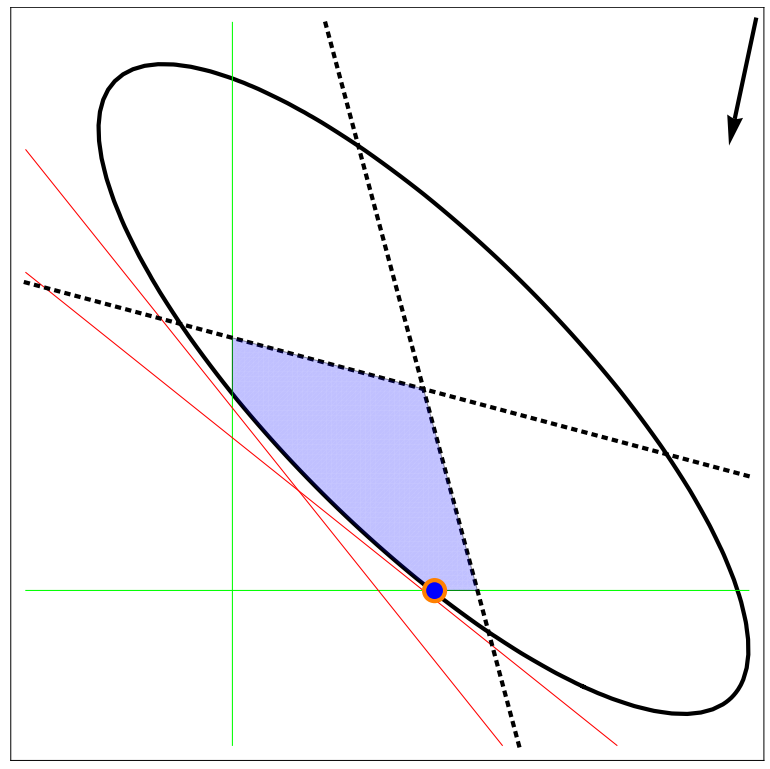

(a) SOCP is exact.

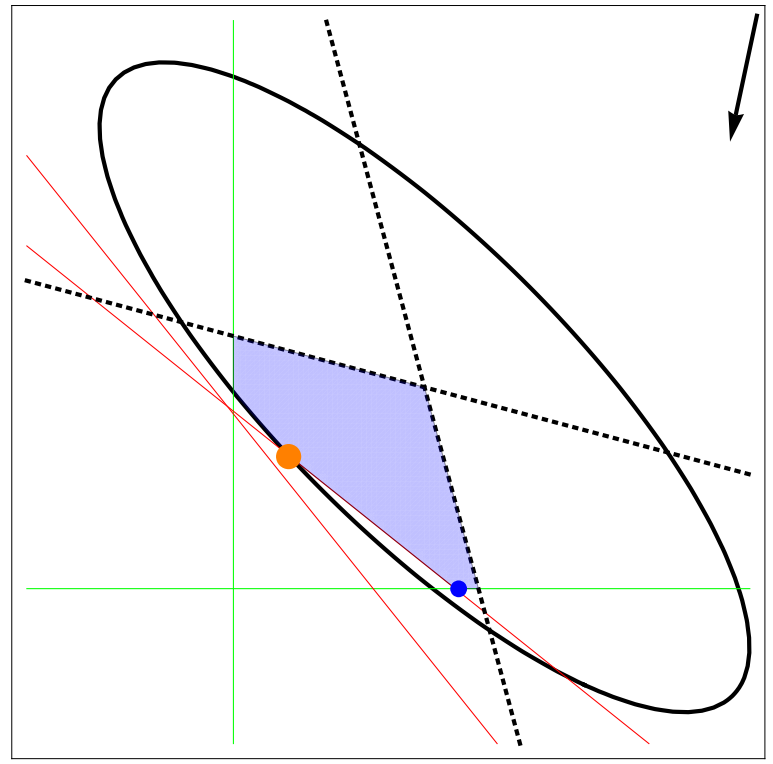

(c) SOCP is inexact due to reactive and active lower bounds.

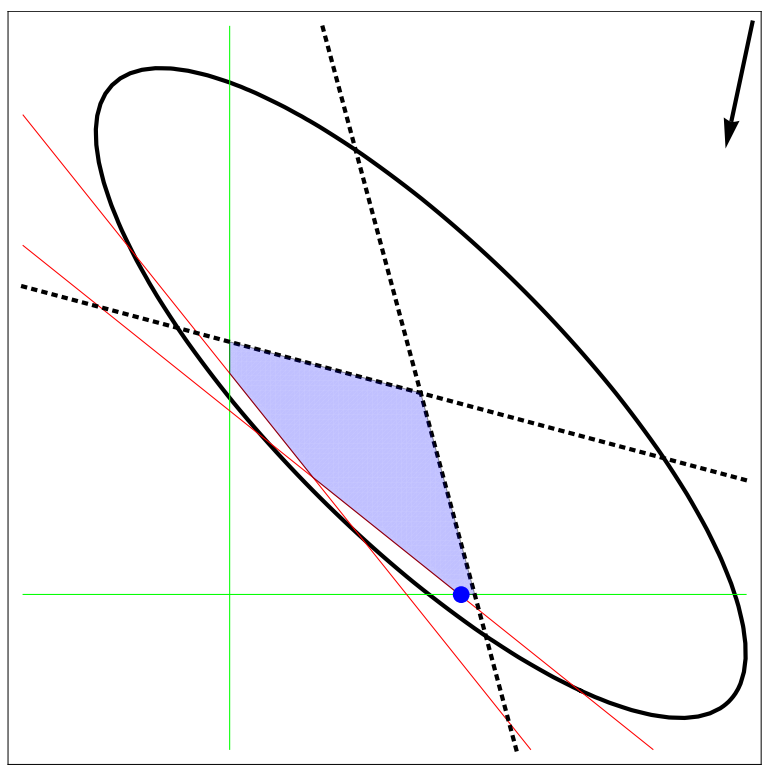

(b) SOCP is feasible while OPF is infeasible.

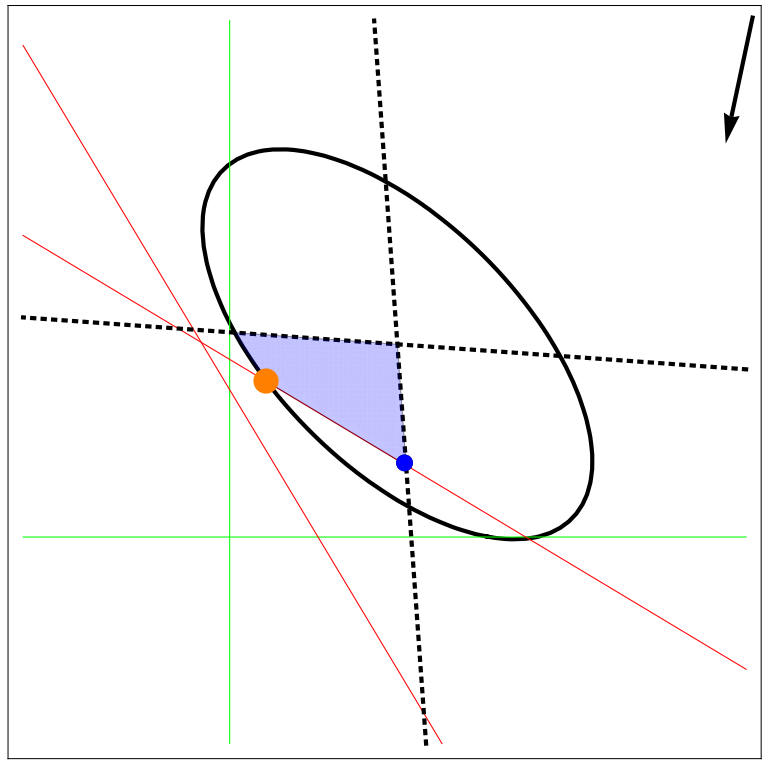

(d) SOCP is inexact due to angle and reactive lower bounds. 
- If $c_{11}^{O}-c_{22}^{O}<\Delta, c_{22}^{E} \geq \underline{c}_{22}$, and $c_{11}^{E} \geq \underline{c}_{11}$, then the SOCP relaxation is exact as in Figure 1c. In fact, any point in the convex combination of $c^{R}$ and $c^{E}$ is SOCP optimal. Such a point can always be corrected by reducing $c_{11}, c_{22}$ and $c_{12}$ components by the same amount until we reach $c^{E}$, which is the OPF optimal solution.

- If $c_{11}^{O}-c_{22}^{O}<\Delta, c_{22}^{E} \geq \underline{c}_{22}$, and $c_{11}^{E}<\underline{c}_{11}$, define the intersection of $c_{11}-c_{22}=\Delta$ with the bounding lower bound of either $c_{11}$ or $c_{22}$ :

$$
\left(c_{11}^{L}, c_{22}^{L}\right)=\left\{\begin{array}{l}
\left(\underline{c}_{22}+\Delta, \underline{c}_{22}\right) \text { if } \underline{c}_{11}-\underline{c}_{22} \leq \Delta \\
\left(\underline{c}_{11}, \underline{c}_{11}-\Delta\right) \text { o.w. }
\end{array}\right.
$$

Observe that any point in the convex combination of $c^{L}$ and $c^{R}$ is SOCP optimal. However, there is no feasible OPF solution with the same objective function value. Lastly, let us define the lower intersection of the hyperbola (10) and the $c_{11}$ lower bound as $\left(c_{11}^{I}, c_{22}^{I}\right)$, where $c_{11}^{I}=\underline{c}_{11}$, and $c_{22}^{I}$ as

$$
c_{22}^{I}=\frac{2 \beta+\underline{c}_{11}-\sqrt{\left(2 \beta+\underline{c}_{11}\right)^{2}-4\left(\alpha^{2}+\beta^{2}\right)}}{2}
$$

We have two final cases:

- If $c_{22}^{I}<\underline{c}_{22}$, any point in the convex combination of $c^{L}$ and $c^{R}$ is SOCP optimal. However, OPF is infeasible. An example of this case can be seen in Figure 1d.

- If $c_{22}^{I} \geq \underline{c}_{22}$, any point in the convex combination of $c^{L}$ and $c^{R}$ is SOCP optimal. However, OPF has a unique optimal solution at $c^{I}$ as can be seen in Figure 1e. Hence, relaxation is inexact. Assuming a linear cost function with coefficient 1, optimality gap can be calculated as $-G\left(c_{22}^{L}-c_{22}^{I}\right)$.

The above analysis proves the following theorem.

Theorem 3.1. In a two-bus one-generator system with linear objective, the SOCP/SDP relaxation of the AC OPF problem has the following possible outcomes:

(i) SOCP relaxation is exact: If $c_{11}^{O}-c_{22}^{O} \geq \Delta$ or if $c_{11}^{O}-c_{22}^{O}<\Delta, c_{22}^{E} \geq \underline{c}_{22}, c_{11}^{E} \geq \underline{c}_{11}$.

(ii) SOCP relaxation is inexact with finite optimality gap: If $c_{11}^{O}-c_{22}^{O}<\Delta, c_{22}^{E} \geq \underline{c}_{22}$, $c_{11}^{E}<\underline{c}_{11}, c_{22}^{I} \geq \underline{c}_{22}$. The optimality gap is $-G\left(c_{22}^{L}-c_{22}^{I}\right)$.

(iii) SOCP relaxation is feasible and OPF is infeasible: If $c_{11}^{O}-c_{22}^{O}<\Delta, c_{22}^{E}<\underline{c}_{22}$ or if $c_{11}^{O}-c_{22}^{O}<\Delta, c_{22}^{E} \geq \underline{c}_{22}, c_{11}^{E}<\underline{c}_{11}, c_{22}^{I}<\underline{c}_{22}$.

Here, $c^{O}, c^{E}, c^{R}, c^{L}, c^{I}$ are defined in (12)-(16), respectively. 


\section{Examples of Inexact SOCP Relaxations}

We have obtained a complete characterization for a 2-bus network with a single generator, and shown that the SOCP relaxation is exact only under certain conditions. In this section, we present further counterexamples of radial networks with two and three buses. Most of the network parameters are selected from IEEE test instances. Transmission line capacity is assumed to be large. For all the buses, $V_{i}^{\min }=0.9$ and $V_{i}^{\max }=1.1$. Production costs are taken as linear functions. OPF problem with alternative formulation (2) is solved to global optimality with BARON [23]. SOCP relaxations are solved using interior point solver MOSEK [1].

\subsection{2-Bus, 2-Generator Example}

Let us consider a 2-bus network with one generator located at each bus. Data of this example is given in Table 1 . The impedance of line $(1,2)$ is $0.01008+\mathrm{i} 0.0504$.

Table 1: Bus and generator data for 2-bus 2-generator example.

\begin{tabular}{rrrrcccr}
\hline Bus & $p_{i}^{d}$ & $q_{i}^{d}$ & $p_{i}^{\min }$ & $p_{i}^{\max }$ & $q_{i}^{\min }$ & $q_{i}^{\max }$ & cost \\
\hline 1 & 75 & -84.7 & 75 & 250 & -30 & 300 & 5.0 \\
2 & 105 & 22.8 & 70 & 300 & -30 & 300 & 1.2 \\
\hline
\end{tabular}

In Table 2, we compare the SOCP relaxation and the global optimal solution of OPF for different levels of load, where load is varied as $\left[\begin{array}{llll}p_{1}^{d} & p_{2}^{d} & q_{1}^{d} & q_{2}^{d}\end{array}\right]=\gamma \cdot\left[\begin{array}{lllll}75 & 105 & -84.7 & 22.8\end{array}\right]$ for some positive parameter $\gamma$.

When $\gamma \in[0.81,0.99]$, we observe that the SOCP relaxation is exact. For $\gamma$ around 1.00, there is a finite optimality gap, which can be as large as $21.44 \%$ at $\gamma=1.01$. Finally, for $\gamma \geq 1.02$, OPF becomes infeasible, whereas SOCP relaxation is still feasible. In fact, SOCP relaxation fails to detect infeasibility of OPF problem until $\gamma$ exceeds 2.93.

Now, let us consider the case where voltages are fixed. In [13], it has been proven that if angle differences are guaranteed to be small enough, then SDP/SOCP relaxations are tight even if there are real power lower bounds. However, we present an example which demonstrates that this does not extend to the case with reactive power lower bounds. To this end, let us fix the squared voltage magnitudes to $\left(c_{11}, c_{22}\right)=(0.874,0.816)$. In this case, the global optimal solution of OPF is 573.82 while the SOCP relaxation gives 503.37. Hence, there is an optimality gap, even though angle difference is less than $1^{\circ}$.

Figure 2 presents possible configurations of the feasible region of the OPF and the SOCP relaxation projected to the $\left(p_{1}^{g}, p_{2}^{g}\right)$ space. In Figure $2 \mathrm{a}$, the SOCP relaxation is exact, while 
Figure 3: Projection of feasible region of 3-bus example onto $\left(p_{1}^{g}, q_{1}^{g}\right)$ space with respect to different load levels. Horizontal axis is $p_{1}^{g}$ and vertical axis is $q_{1}^{g}$. Black curve and blue region are the feasible regions of OPF and SOCP relaxation, respectively. Red line is the lower bound on $q_{1}^{g}$. All figures are in p.u.
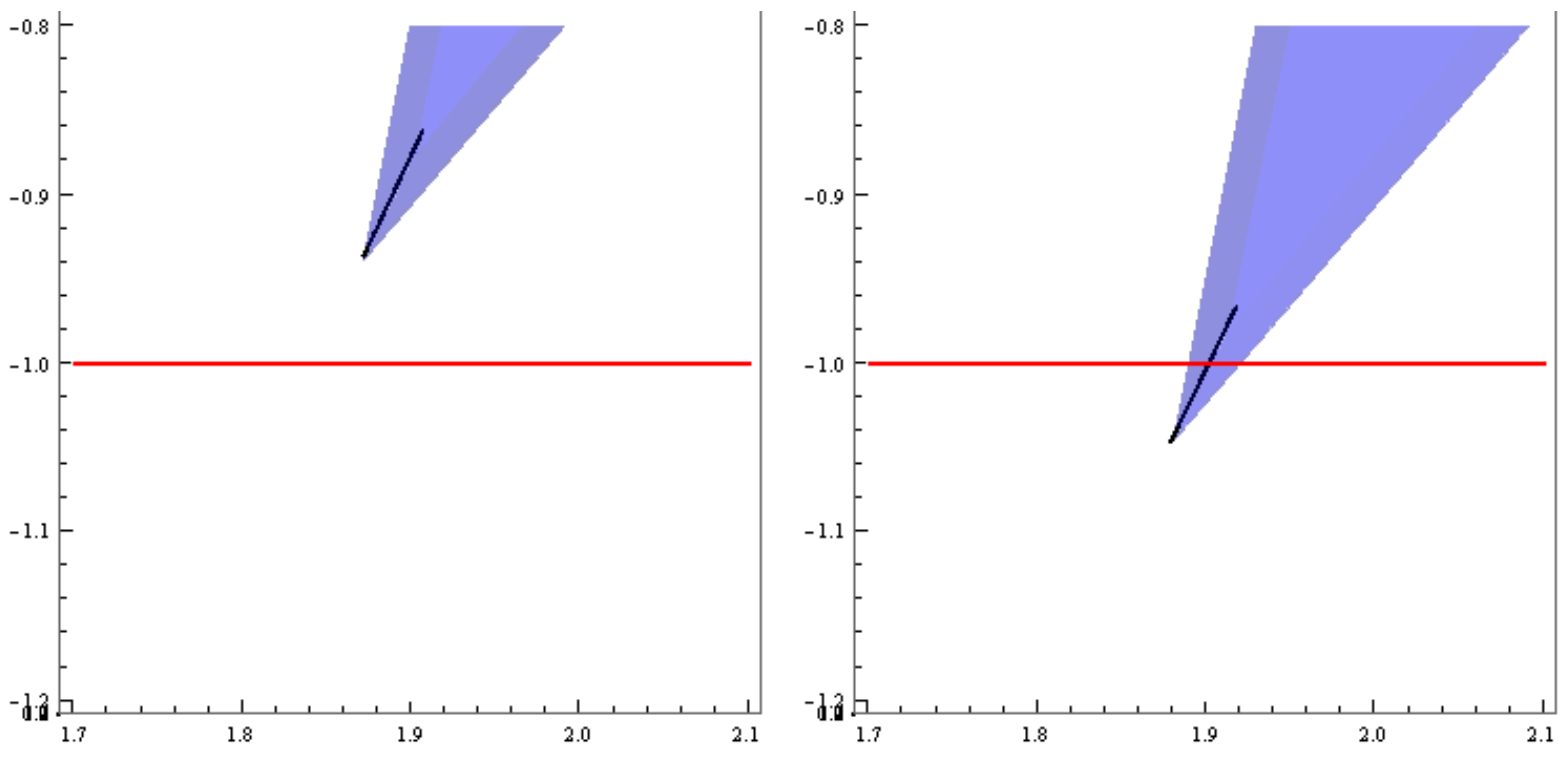

(a) $\gamma=0.90$

(b) $\gamma=1.00$

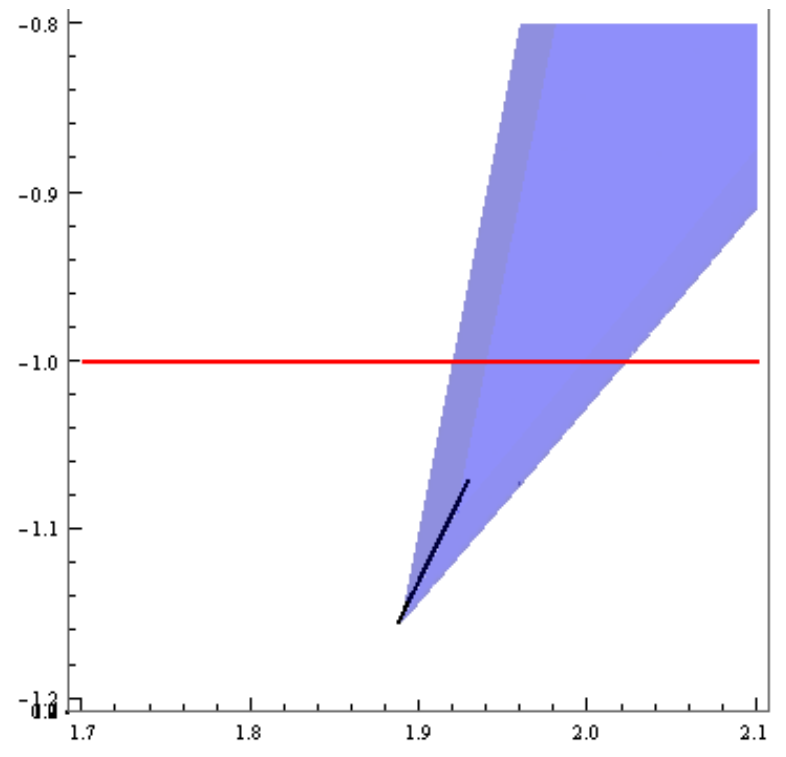

(c) $\gamma=1.10$ 
Table 2: Objective costs for 2-bus 2-generator with varying load.

\begin{tabular}{rrr}
\hline$\gamma$ & OPF & SOCP \\
\hline 0.12 & infeasible & infeasible \\
0.13 & infeasible & 459.00 \\
0.80 & infeasible & 459.00 \\
0.81 & 460.13 & 460.13 \\
0.98 & 496.96 & 496.96 \\
0.99 & 499.15 & 499.15 \\
1.00 & 563.56 & 501.46 \\
1.01 & 641.21 & 503.76 \\
1.02 & infeasible & 506.07 \\
2.92 & infeasible & 1608.75 \\
2.93 & infeasible & infeasible \\
\hline
\end{tabular}

in Figure 2b, the OPF is infeasible although the SOCP is feasible. In Figure 2c, the SOCP relaxation is inexact due to the combined effect of active and reactive lower bounds. Finally, in Figure 2d, the SOCP relaxation is inexact due to practical angle bounds $\left(30^{\circ}\right)$ and reactive lower bounds.

\subsection{3-Bus, 1-Generator Example}

Consider a 3-bus radial network with three loads $\left[\begin{array}{lll}p_{1}^{d} & p_{2}^{d} & p_{3}^{d}\end{array}\right]=\left[\begin{array}{lll}50 & 70 & 60\end{array}\right]$ and $\left[\begin{array}{lll}q_{1}^{d} & q_{2}^{d} & q_{3}^{d}\end{array}\right]=$ $\left[\begin{array}{lll}-52.3 & 14.1 & -82.3\end{array}\right]$. The impedance of lines $(1,2)$ and $(2,3)$ are $0.01008+\mathrm{i} 0.0504$ and $0.07500+\mathrm{i} 0.0840$. The only generator is located at bus 1 with $150 \leq p_{1}^{g} \leq 550$ and $-100 \leq$ $q_{1}^{g} \leq 500$. The cost of power generation is $\$ 5$ per MW. Assume that the reactive load is scaled as $\left[\begin{array}{lll}q_{1}^{d} & q_{2}^{d} & q_{3}^{d}\end{array}\right]=\gamma\left[\begin{array}{lll}-52.3 & 14.1 & -82.3\end{array}\right]$ for some positive $\gamma$. Table 3 shows the optimal costs of the OPF and the SOCP relaxation for different values of $\gamma$.

Table 3: Objective costs for 3-bus example with varying load.

\begin{tabular}{rrr}
\hline$\gamma$ & OPF & SOCP \\
\hline 0.95 & 939.45 & 939.45 \\
0.96 & 939.90 & 939.90 \\
0.97 & 941.57 & 940.87 \\
1.00 & 950.70 & 945.45 \\
1.03 & 959.91 & 950.05 \\
1.04 & infeasible & 951.60 \\
\hline
\end{tabular}


For small values of $\gamma$, e.g. $\gamma \leq 0.96$, SOCP is exact. For values around $\gamma=1$, we observe a finite optimality gap between OPF and SOCP, where for larger values of $\gamma \geq 1.04$, the OPF becomes infeasible while the SOCP relaxation is still feasible. The infeasibility is exactly caused by the lower bound on reactive generation power.

For this example, we also give the feasible region of OPF problem projected onto the $\left(p_{1}^{g}, q_{1}^{g}\right)$ space in Figure 3. When $\gamma=0.90$, reactive power lower bound is redundant and the optimal solution of SOCP relaxation is feasible for OPF. However, for $\gamma=1.00$, constraint

$q_{1}^{\min } \leq q_{1}^{g}$ is binding. Note that the optimal solution of the SOCP relaxation is not feasible for OPF and hence, the relaxation is not exact. Finally, when $\gamma=1.10$, SOCP is feasible whereas OPF is infeasible.

\section{Library of Radial Networks with Inexact SDP/SOCP Relaxation}

\subsection{Generation of Instances}

To facilitate further research, we generate several radial network instances from meshed networks in MATPOWER [27]. Given a meshed network, we first find a spanning tree by switching off lines to obtain a radial network. Then, only load values and generation lower/upper bounds are changed, whenever necessary, to construct examples where the SOCP/SDP relaxation is not exact. New instances can be downloaded from https: //sites.google.com/site/burakkocuk/.

Our examples are based on 9-, 14-, 30-, 39- and 57-bus standard instances. Due to our construction of the network topology, AC feasibility becomes a major issue. Although unrealistic examples can be constructed for even larger networks by reducing load values considerably, we choose not to sacrifice the realistic features of the instances.

\subsection{Computational Results for SDP Relaxation vs. Global Optimal Solution}

For each instance generated as described above, we solve the SDP relaxation using MOSEK [1]. The code is written in C\# language and Visual Studio 2010 is used as the compiler. We report the value of the objective function, computation time and the rank of the solution. Here, rank is determined as the number of eigenvalues that are larger than $10^{-5}$.

SDP relaxation is compared against global optimal solution found using BARON [23] and local solution found by MATPOWER [27] and IPOPT [25]. Relative optimality gap for BARON is set to 0 so that global optimality can be certified. We should note that

performance of BARON on rectangular formulation (1) is very poor as it requires hours to prove global optimality. Instead, we use reformulation (2), which is valid for radial networks. 
Table 4: SDP relaxation vs. global solver BARON and local solver MATPOWER.

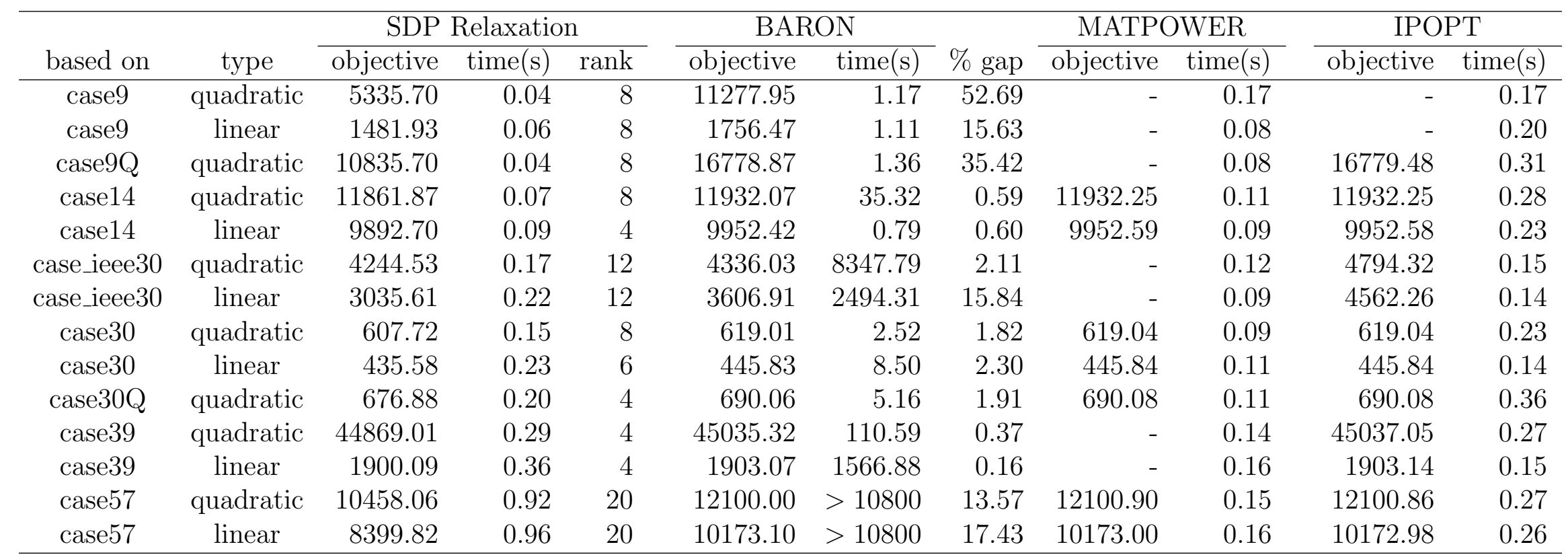


For all experiments, we used a 64-bit computer with Intel Core i5 CPU 3.33GHz processor and 4 GB RAM. Each instance is solved twice with quadratic and linear objectives. For the latter, we simply ignore the quadratic cost coefficients.

Our findings are summarized in Table 4. One can see that the SDP relaxation solution can be of high-rank (up to 12 for case_ieee30 and 20 for case57). Also, the optimality gap (column "\% gap") computed as $100 \times\left(1-z^{S D P} / z^{B A R O N}\right)$, where $z^{S D P}$ and $z^{B A R O N}$ are respectively the values of the SDP relaxation and the global optimal solution found by BARON, can be quite large (more than $52 \%$ for case9 with quadratic objective). Our examples clearly show that the optimal value of the SDP relaxation can be quite different from the global optimal value. We also compare the optimal dispatch solutions $p^{S D P}$ and $p^{B A R O N}$ computed by the SDP relaxation and BARON to show that large differences in the objective function values are not artifacts of the cost parameters. In fact, the 2 -norm $\left\|p^{S D P}-p^{B A R O N}\right\|$ is large, varying from 0.16 p.u. to 3.16 p.u. for our instances. This illustrates that the optimal solutions are quite different from one another.

In general, MATPOWER is accepted to be a reliable and efficient OPF solver. It manages to find the global optimal solution up to a negligible difference for seven of the instances from our library. However, we observe that it fails to solve the remaining seven instances due to numerical issues. There are other robust NLP solvers available, e.g. IPOPT, which gives near global optimal solution for nine instances in the library, where small discrepancies in optimal objective function values compared to BARON are due to numerical errors. On the other hand, IPOPT fails in two 9-bus examples and it finds suboptimal solutions three times for both of the case_ieee30 instances and case39 with quadratic objective.

We should note that the global solver BARON can be computationally expensive. For instance, for case_ieee30 with a quadratic objective, it requires more than 2 hours to prove optimality whereas for 57-bus instances, BARON is not able to certify the global optimal solution within 3 hours time limit. Upon termination, the optimality gaps are $38.40 \%$ and $29.37 \%$ for quadratic and linear objectives, respectively. Also, the reformulation of OPF (2) is only valid for radial networks. Hence, in general, using BARON as it is may not be applicable to large-scale OPFs.

\section{Bound Tightening and Valid Inequalities for Global Optimization}

In this section, we propose valid inequalities for the SOCP relaxation of the OPF problem to improve the computational time of the global solver BARON. The main algorithm of BARON is based on spatial branch-and-bound [23]. It utilizes convex envelopes of the feasible region and polyhedral relaxations to improve lower bounds and prove global optimality. Therefore, 
it is very important to add valid inequalities and variable bounds so that BARON can obtain tighter relaxations. A more detailed description is provided in Appendix .1.

To begin with, let us focus on formulation (2). Observe that $c_{i j}$ and $s_{i j}$ do not have explicit variable bounds although they have implied bounds due to (2d) and (2i) as

$$
-V_{i}^{\max } V_{j}^{\max } \leq c_{i j}, s_{i j} \leq V_{i}^{\max } V_{j}^{\max } \quad(i, j) \in \mathcal{L}
$$

However, these bounds are very loose knowing that angle differences are generally small. This fact suggests that these bounds can be improved. One way to obtain variable bounds is to optimize $c_{i j}$ and $s_{i j}$ over the set $\mathcal{S}=\{(p, q, c, s):(2 b)-(2 i)\}$, which is a nonconvex set. Alternatively, one can find weaker bounds over the set $\mathcal{S}^{\prime}=\{(p, q, c, s):(2 b)-(2 h),(5)\}$ by solving SOCP relaxations. Let $\underline{c}_{i j}\left(\underline{s}_{i j}\right)$ and $\bar{c}_{i j}\left(\bar{s}_{i j}\right)$ denote lower and upper bounds found for $c_{i j}\left(s_{i j}\right)$, respectively.

Now, let us investigate how the box $\mathcal{B}_{i j}=\left[\underline{c}_{i j}, \bar{c}_{i j}\right] \times\left[\underline{s}_{i j}, \bar{s}_{i j}\right]$ is positioned with respect

to the "ring"-like set $\mathcal{R}_{i j}=\left\{\left(c_{i j}, s_{i j}\right): \underline{R}_{i j}^{2} \leq c_{i j}^{2}+s_{i j}^{2} \leq \bar{R}_{i j}^{2}\right\}$ where $\underline{R}_{i j}=V_{i}^{\min } V_{j}^{\min }$ and $\bar{R}_{i j}=V_{i}^{\max } V_{j}^{\max }$. In our experiments, we observe that $\underline{c}_{i j}>0$, which we assume hereafter. We should note that this is not a restrictive assumption, similar valid inequalities described below can be generated even if this assumption does not hold.

Let us focus on the case with $\underline{c}_{i j}<\underline{R}_{i j}$, which gives rise to four possibilities:

- Case 1: $\left\|\left(\underline{c}_{i j}, \underline{s}_{i j}\right)\right\|<\underline{R}_{i j},\left\|\left(\underline{c}_{i j}, \bar{s}_{i j}\right)\right\|<\underline{R}_{i j}$

- Case 2: $\left\|\left(\underline{c}_{i j}, \underline{s}_{i j}\right)\right\|<\underline{R}_{i j},\left\|\left(\underline{c}_{i j}, \bar{s}_{i j}\right)\right\| \geq \underline{R}_{i j}$

- Case 3: $\left\|\left(\underline{c}_{i j}, \underline{s}_{i j}\right)\right\| \geq \underline{R}_{i j},\left\|\left(\underline{c}_{i j}, \bar{s}_{i j}\right)\right\|<\underline{R}_{i j}$

- Case 4: $\left\|\left(\underline{c}_{i j}, \underline{s}_{i j}\right)\right\| \geq \underline{R}_{i j},\left\|\left(\underline{c}_{i j}, \bar{s}_{i j}\right)\right\| \geq \underline{R}_{i j}$

Figure 4 shows typical examples for each of four cases. In the rest of this section, we concentrate on how we can obtain valid inequalities for Cases 1, 2, and 3.

\subsection{Valid Inequalities}

These cuts are designed to cut off the portion of $\mathcal{B}_{i j}$ inside the inner circle for Cases 1, 2, and 3 as depicted in Figure 4. Algorithm 1 gives the exact procedure. Note that the validity of the inequality follows from the fact that the points cut off from the box have norm less than $\underline{R}_{i j}$. Note that for Case 4 , the algorithm would produce the inequality $c_{i j} \geq \underline{c}_{i j}$, hence it is omitted. 
Figure 4: Positioning of $\mathcal{B}_{i j}$ and $\mathcal{R}_{i j}$. Red line is the cut produced by Algorithm 1, when applicable.

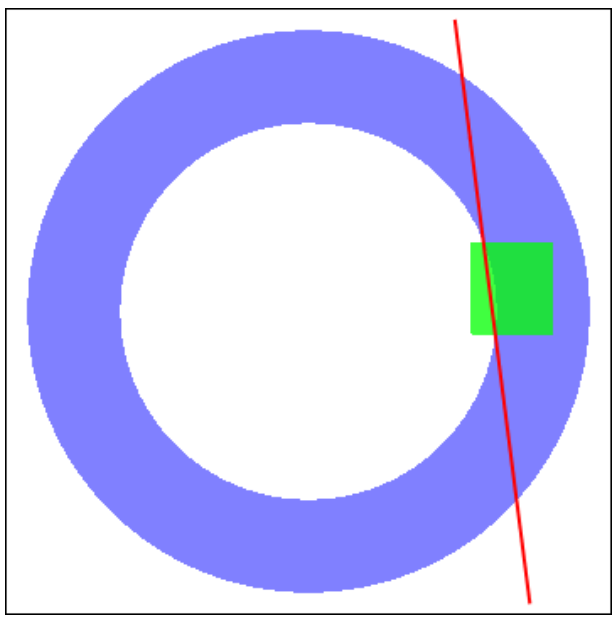

(a) Case 1

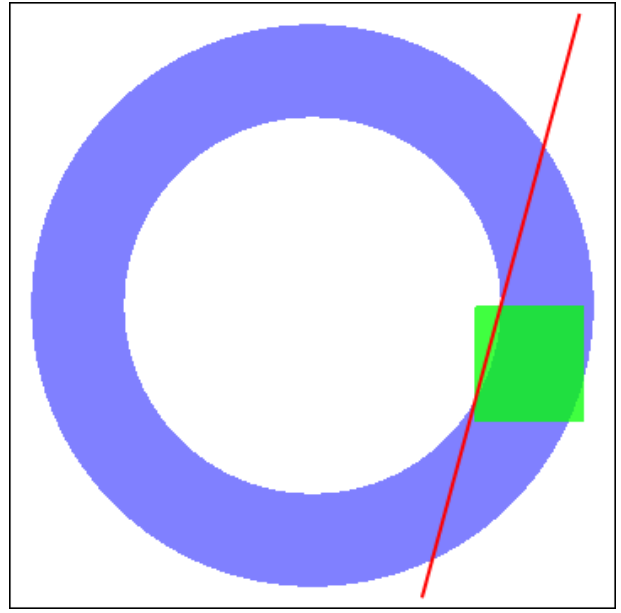

(c) Case 3

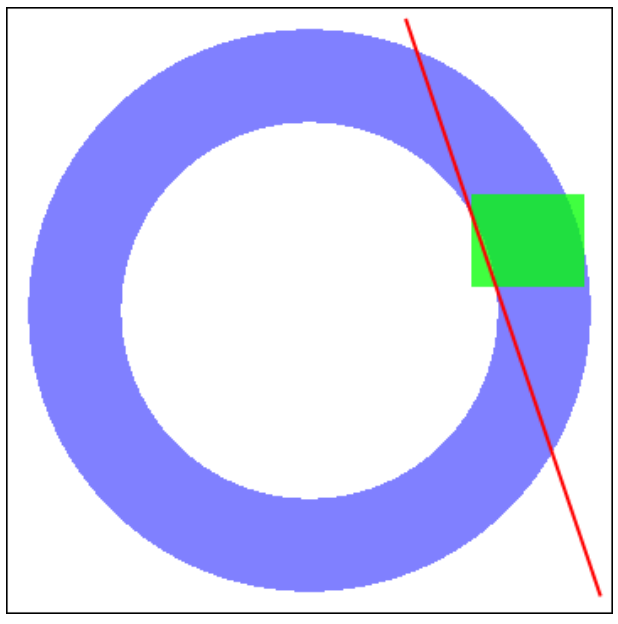

(b) Case 2

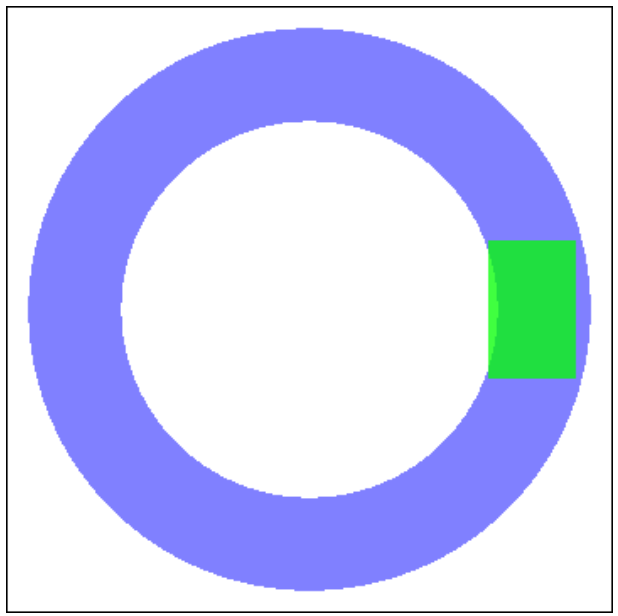

(d) Case 4 


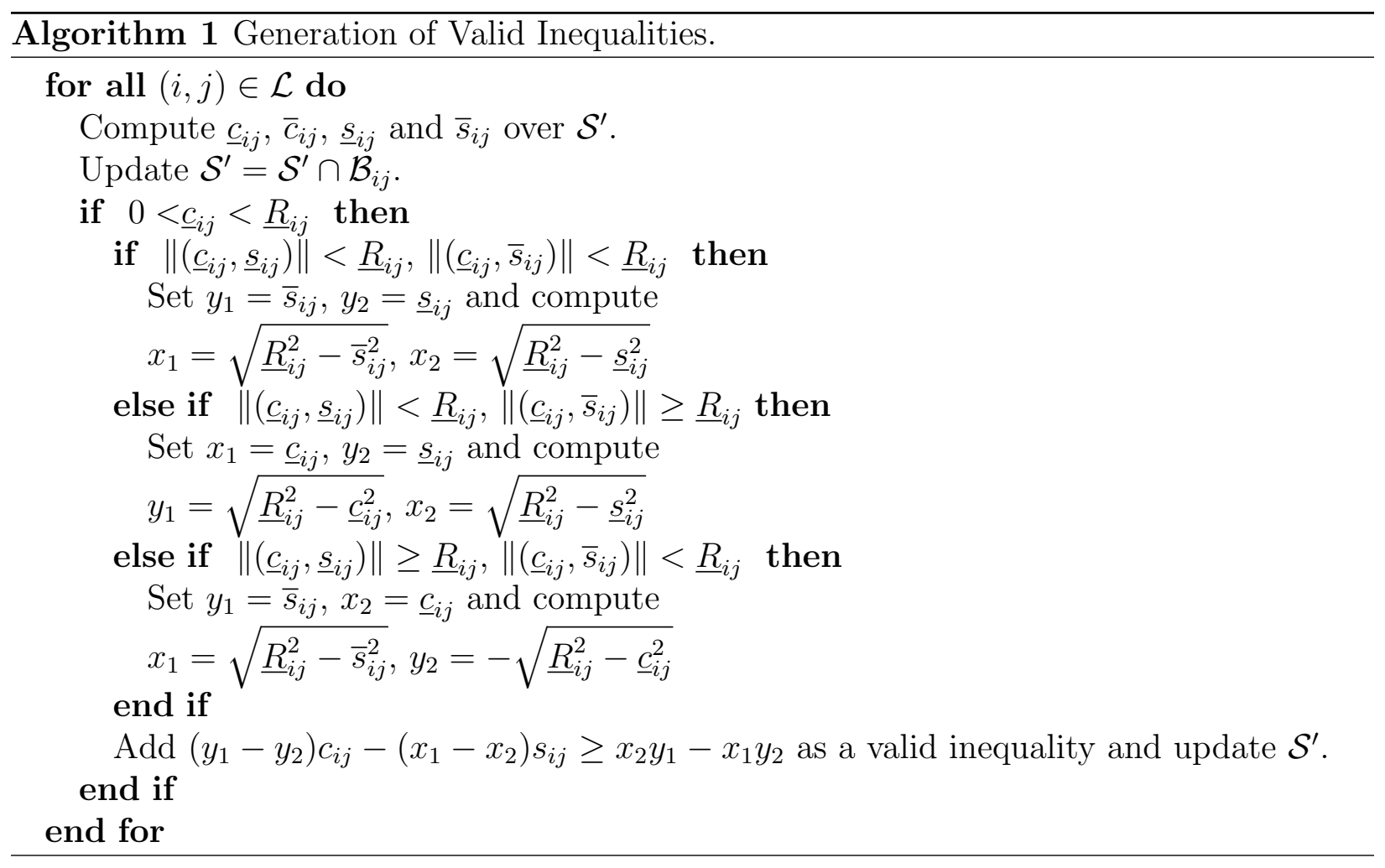

\subsection{Numerical Experiments}

The effect of valid inequalities are tested on our library of instances. The results are summarized in Table 5. We should note that MATPOWER is very efficient and accurate for the seven instances it is able to solve as shown in Table 4. Therefore, we mainly focus on the other seven instances where MATPOWER fails to solve. In Table 5, preprocessing refers to computing variable bounds and valid cuts.

For the 9-bus instances (case9, case9Q), BARON's computation time reduces slightly with the addition of cuts. However, the preprocessing time dominates the total computation time, which is larger than the case without cuts.

For the 30-bus IEEE instances, BARON can require hours to terminate. With the addition of variable bounds, total computation time reduces by $89 \%$ and $90 \%$ for quadratic and linear objectives, respectively. Quite impressively, the inclusion of valid inequalities further reduces the total computation time to only 17 seconds, less than $0.1 \%$ of the computation time without variable bounds and cuts.

For 39-bus instances, the addition of variable bounds brings down total computation time by $76 \%$ and $95 \%$ for quadratic and linear objectives, respectively. In this case, the inclusion of valid inequalities decreases the computational time for linear objective. On the other hand, cuts slightly increases the total computational time in the case of quadratic objective. 
Table 5: BARON with bounds and cuts. PT, BT and TT represent times of preprocessing, BARON solution and total computation in seconds. $\mathrm{RG}$ represents the percentage root gap.

\begin{tabular}{|c|c|c|c|c|c|c|c|c|c|c|c|c|}
\hline \multirow{2}{*}{ based on } & \multirow[b]{2}{*}{ type } & \multicolumn{2}{|c|}{ BARON } & \multicolumn{4}{|c|}{ BARON with bounds } & \multicolumn{5}{|c|}{ BARON with bounds and cuts } \\
\hline & & $\mathrm{BT}(\mathrm{s})$ & $\mathrm{RG}(\%)$ & $\mathrm{PT}(\mathrm{s})$ & $\mathrm{BT}(\mathrm{s})$ & $\mathrm{TT}(\mathrm{s})$ & $\mathrm{RG}(\%)$ & $\mathrm{PT}(\mathrm{s})$ & $\mathrm{BT}(\mathrm{s})$ & $\mathrm{TT}(\mathrm{s})$ & \#cuts & $\mathrm{RG}(\%)$ \\
\hline case 9 & quadratic & 1.17 & 11.72 & 4.34 & 1.08 & 5.42 & 9.72 & 4.41 & 1.01 & 5.42 & 6 & 9.71 \\
\hline case9 & linear & 1.11 & 16.13 & 4.12 & 0.86 & 4.98 & 16.02 & 4.42 & 1.00 & 5.42 & 6 & 16.05 \\
\hline case9Q & quadratic & 1.36 & 16.91 & 4.36 & 1.22 & 5.58 & 8.32 & 4.34 & 1.11 & 5.46 & 6 & 7.50 \\
\hline case14 & quadratic & 35.32 & 9.98 & 7.11 & 30.46 & 37.56 & 1.98 & 6.89 & 41.99 & 48.88 & 7 & 1.98 \\
\hline case14 & linear & 0.79 & 0.21 & 6.95 & 0.83 & 7.79 & 0.15 & 6.89 & 0.91 & 7.80 & 7 & 0.39 \\
\hline case_ieee30 & quadratic & 8347.79 & 46.93 & 16.91 & 900.50 & 917.41 & 29.88 & 17.28 & 0.36 & 17.63 & 14 & 0.00 \\
\hline case_ieee30 & linear & 2494.31 & 46.67 & 16.89 & 249.48 & 266.37 & 33.69 & 16.96 & 0.34 & 17.30 & 14 & 0.00 \\
\hline case30 & quadratic & 2.52 & 9.13 & 17.21 & 1.91 & 19.12 & 7.74 & 16.94 & 4.42 & 21.35 & 13 & 7.39 \\
\hline case30 & linear & 8.50 & 5.79 & 17.60 & 2.40 & 19.99 & 5.15 & 16.23 & 1.91 & 18.14 & 13 & 4.41 \\
\hline case30Q & quadratic & 5.16 & 13.25 & 16.53 & 2.39 & 18.93 & 12.11 & 16.80 & 1.83 & 18.64 & 13 & 4.27 \\
\hline case39 & quadratic & 110.59 & 8.89 & 28.07 & 26.03 & 54.10 & 0.48 & 27.72 & 33.25 & 60.98 & 12 & 1.12 \\
\hline case39 & linear & 1566.88 & 2.56 & 26.94 & 72.80 & 99.74 & 0.51 & 28.17 & 42.62 & 70.79 & 12 & 0.52 \\
\hline case57 & quadratic & $>10800$ & 46.69 & 41.57 & 0.66 & 42.23 & 0.00 & 40.17 & 0.80 & 40.97 & 14 & 0.00 \\
\hline case57 & linear & $>10800$ & 45.17 & 42.19 & 0.67 & 42.87 & 0.00 & 45.23 & 0.67 & 45.91 & 14 & 0.00 \\
\hline
\end{tabular}


However, compared to the case without bounds and cuts, BARON still requires less amount of time.

For 57-bus instances, BARON without bounds was not able to certify the global optimal solution within 3 hours time limit. However, the strengthened variable bounds and valid inequalities enable BARON to solve these instances to global optimality within only 46 seconds.

As a final note, we should note that the applicability of the valid inequalities proposed in this section is not limited to the global optimization of radial networks, they can be used in meshed networks as well. Moreover, precisely the same valid inequalities can be used in SOCP relaxation whereas the transformations $c_{i j}=e_{i} e_{j}+f_{i} f_{j}$ and $s_{i j}=e_{i} f_{j}-e_{j} f_{i}$ enable us to obtain linear matrix inequalities to be added to SDP relaxation. Although, for our instances, we have not observed any lower bound improvement in SOCP/SDP relaxations by the inclusion of the valid inequalities, we obtain stronger root node relaxations in BARON. Let $\mathrm{RG}$ represent the percentage root gap calculated as $100 \times\left(1-z_{\mathrm{r}} / z_{\mathrm{g}}\right)$, where $z_{\mathrm{r}}$ and $z_{\mathrm{g}}$ are respectively the values of root node relaxation for BARON and global optimal solution. As we can see from Table 5 that addition of bounds and valid inequalities strengthen the root node relaxation of BARON in general. In fact, case_ieee30 and case57 instances are already solved at the root node. We should note that occasionally RG of BARON with bounds and cuts is slightly worse than BARON with bounds. However, this is due to the fact that valid inequalities change the problem structure and may lead to different preprocessing procedures carried out by the solver at the root node.

\section{Conclusions}

In this work, we study the impact of generation lower bounds on the performance of convex relaxations of $\mathrm{AC} \mathrm{OPF}$ problems. For the fundamental two-bus one-generator model, we provide a complete characterization of all possible outcomes of the SOCP relaxation together with a detailed study of the projected feasible regions of the OPF and SOCP relaxation. We provide a library of radial network instances that demonstrate large optimality gaps for SDP and SOCP relaxations. We also propose valid inequalities for the SOCP relaxation, which prove to be useful in reducing the computation time of global solver BARON. We remind the reader here that SDP relaxations are very powerful and their importance is definite. Our work only serves to demonstrate the limitations of SDP relaxations and emphasizes the importance and the need to develop efficient global methods in solving OPF problems. 


\section{.1 A Note on BARON}

Branch-and-Reduce Optimization Navigator (BARON) is a general purpose global solver designed to solve Mixed-Integer Nonlinear Programs (MINLP). The details of the algorithm can be found in [24]. Here, we only describe the key elements in the algorithm.

Given an MINLP, BARON first transforms the problem into a factorable form [18] using compositions of sums and products of single-variable functions. This form enables the solver to find polyhedral outer approximations of the feasible region using McCormick envelopes or other envelopes, depending on the type of the nonlinearity. After this step, linear programs are used to solve the convex relaxations. Also, NLP solvers are utilized to find feasible solutions, which serves as upper bounding heuristics.

There are two features in the algorithm which help to refine the convex relaxation by updating bounds on the variables. First of these features is called "range reduction". If a variable is at its upper bound in a relaxed subproblem, its lower bound may be improved. In case reduction step fails, then the second feature called "spatial branching" is applied. In particular, a variable is selected and bisection is done to create two new nodes to be explored with new bounds on the branched variable. The algorithm terminates when either all nodes are explored or lower bound and upper bound are close enough to a certain degree, which is predefined by the user.

As this brief explanation demonstrates, the success of BARON heavily depends on the quality of convex relaxations. In particular, good variable bounds are crucial in order to obtain tight polyhedral relaxations. This will help the solver to limit the number of branching steps and reduce the depth of search tree. Similarly, problem-specific valid inequalities can be useful to tighten the convex relaxation.

\section{References}

[1] MOSEK Modeling Manual. MOSEK ApS, 2013.

[2] Subhonmesh Bose, Dennice F Gayme, K Mani Chandy, and Steven H Low. Quadratically constrained quadratic programs on acyclic graphs with application to power flow. arXiv preprint arXiv:1203.5599, 2012.

[3] Subhonmesh Bose, Dennice F Gayme, Steven Low, and K Mani Chandy. Optimal power flow over tree networks. In 49th Annual Allerton Conference on Communication, Control, and Computing (Allerton), pages 1342-1348, 2011. 
[4] Waqquas A. Bukhsh, Andreas Grothey, Ken McKinnon, and Paul Trodden. Local solutions of optimal power flow. IEEE Trans. on Power Syst., 28(4):4780 - 4788, 2013.

[5] J. Carpentier. Contributions to the economic dispatch problem. Bulletin Society Francaise Electriciens, 8(3):431-447, 1962.

[6] A. G. Expósito and E. R. Ramos. Reliable load flow technique for radial distribution networks. IEEE Trans. on Power Syst., 14(3):1063 - 1069, 1999.

[7] Lingwen Gan, Na Li, Steven Low, and Ufuk Topcu. Exact convex relaxation for optimal power flow in distribution networks. SIGMETRICS Perform. Eval. Rev., 41(1):351-352, June 2013.

[8] Rabih A. Jabr. Radial distribution load flow using conic programming. IEEE Trans. Power Syst., 21(3):1458-1459, 2006.

[9] Rabih A. Jabr. A conic quadratic format for the load flow equations of meshed networks. IEEE Trans. Power Syst., 22(4):2285-2286, 2007.

[10] Rabih A. Jabr. Optimal power flow using an extended conic quadratic formulation. IEEE Trans. Power Syst., 23(3):1000-1008, 2008.

[11] Cédric Josz, Jean Maeght, Patrick Panciatici, and Jean Charles Gilbert. Application of the moment-sos approach to global optimization of the opf problem. IEEE Trans. on Power Syst., 2014.

[12] Jean B Lasserre. Global optimization with polynomials and the problem of moments. SIAM Journal on Optimization, 11(3):796-817, 2001.

[13] J. Lavaei, D. Tse, and Baosen Zhang. Geometry of power flows and optimization in distribution networks. IEEE Trans. Power Syst., 29(2):572-583, March 2014.

[14] Javad Lavaei and Steven H. Low. Zero duality gap in optimal power flow problem. IEEE Trans. on Power Syst., 27(1):92-107, 2012.

[15] Bernard C. Lesieutre, Daniel K. Molzhan, Alex R. Borden, and Christopher L. DeMarco. Examining the limits of the application of semidefinite programming to power flow problems. In Forty-Nine Annual Allerton Conference, pages 1492-1499, 2011.

[16] Steven H Low. Convex relaxation of optimal power flow, part i: Formulations and equivalence. IEEE Trans. Control Netw. Syst., 1(1):15-27, March 2014. 
[17] Steven H Low. Convex relaxation of optimal power flow, part ii: Exactness. IEEE Trans. Control Netw. Syst., 1(2):177-189, June 2014.

[18] Garth P McCormick. Computability of global solutions to factorable nonconvex programs: Part I - convex underestimating problems. Mathematical Programming, 10(1):147-175, 1976.

[19] Dzung T. Phan. Lagrangian duality and branch-and-bound algorithms for optimal power flow. Operations Research, 60(2):275-285, 2012.

[20] Somayeh Sojoudi and Javad Lavaei. Physics of power networks makes hard optimization problems easy to solve. In IEEE Power and Energy Society General Meeting, pages 1-8, 2012.

[21] Somayeh Sojoudi and Javad Lavaei. On the exactness of semidefinite relaxation for nonlinear optimization over graphs: Part ii. In IEEE 52nd Annual Conference on Decision and Control (CDC), pages 1051-1057, Dec 2013.

[22] Andy Sun and Dzung T. Phan. Wiley Encyclopedia of Operations Research and Management Science, chapter Some Optimization Models and Techniques for Electric Power System Short-term Operations. John Wiley \& Sons, Inc., 2013.

[23] M. Tawarmalani and N. V. Sahinidis. A polyhedral branch-and-cut approach to global optimization. Mathematical Programming, 103(2):225-249, 2005.

[24] Mohit Tawarmalani and Nikolaos V Sahinidis. Convexification and Global Optimization in Continuous and Mixed-Integer Nonlinear Programming: Theory, Algorithms, Software, and Applications, volume 65. Springer, 2002.

[25] Andreas Wächter and Lorenz T Biegler. On the implementation of an interior-point filter line-search algorithm for large-scale nonlinear programming. Mathematical Programming, 106(1):25-57, 2006.

[26] Baosen Zhang and D. Tse. Geometry of feasible injection region of power networks. In Communication, Control, and Computing (Allerton), 2011 49th Annual Allerton Conference on, pages 1508-1515, Sept 2011.

[27] R.D. Zimmerman, C.E. Murillo-Sanchez, and R.J. Thomas. MATPOWER: Steady-state operations, planning, and analysis tools for power systems research and education. IEEE Trans. Power Syst., 26(1):12-19, Feb 2011. 